\title{
Mps1 is SUMO-modified during the cell cycle
}

\author{
Agnese Restuccia ${ }^{1, *}$, Feikun Yang ${ }^{2,}{ }^{*}$, Changyan Chen ${ }^{3}$, Lou Lu ${ }^{4}$, Wei Dai ${ }^{2}$ \\ ${ }^{1}$ Division of Virus-Associated Carcinogenesis, German Cancer Research Center, Heidelberg, Germany \\ ${ }^{2}$ Departments of Environmental Medicine, Biochemistry and Molecular Pharmacology, New York University Langone Medical \\ Center, Tuxedo Park, NY, USA \\ ${ }^{3}$ Center for Drug Discovery, Northeastern University, Boston, MA, USA \\ ${ }^{4}$ Division of Molecular Medicine, Department of Medicine, David Geffen School of Medicine, University of California Los \\ Angeles, Torrance, CA, USA \\ *These authors contributed equally to this work
}

Correspondence to: Wei Dai, e-mail: wei.dai@nyumc.org

Keywords: Mps1, mitosis, sumoylation, BubR1

Received: August 24, 2015

Accepted: November 21, 2015

Published: December 10, 2015

\section{ABSTRACT}

Mps1 is a dual specificity protein kinase that regulates the spindle assembly checkpoint and mediates proper microtubule attachment to chromosomes during mitosis. However, the molecular mechanism that controls Mps1 protein level and its activity during the cell cycle remains unclear. Given that sumoylation plays an important role in mitotic progression, we investigated whether Mps1 was SUMOmodified and whether sumoylation affects its activity in mitosis. Our results showed that Mps1 was sumoylated in both asynchronized and mitotic cell populations. Mps 1 was modified by both SUMO-1 and SUMO-2. Our further studies revealed that lysine residues including K71, K287, K367 and K471 were essential for Mps1 sumoylation. Sumoylation appeared to play a role in mediating kinetochore localization of Mps1, thus affecting normal mitotic progression. Furthermore, SUMO-resistant mutants of Mps1 interacted with BubR1 more efficiently than it did with the wild-type control. Combined, our results indicate that Mps1 is SUMO-modified that plays an essential role in regulating Mps1 functions during mitosis.

\section{INTRODUCTION}

The spindle assembly checkpoint (SAC) is a conserved surveillance mechanism that regulates partitioning of duplicated genome into two daughter cells during mitosis [1]. Extensive research in the past has revealed that protein kinases including Aurora A/B, Mps1, Bub1, and BubR1 positively regulate SAC activities, thus arresting cell at metaphase until all condensed chromosomes are correctly orientated $[2,3]$. The key molecular target of SAC is Cdc20, a substrate-specific activator of anaphase promoting complex/cyclosome (APC/C) [4]. Cdc20, Mad2, Mad3/BubR1, and Bub3 form the mitotic checkpoint complex (MCC), inhibiting the ubiquitin $\mathrm{E} 3$ ligase activity of APC/C [5, 6]. Increasing evidence indicates that $\mathrm{SAC}$ components are promising target for cancer drug development $[7,8]$.

Mps1 (mono-polar spindle 1) is an evolutionarily conserved protein that functions as a key component of SAC $[9,10]$. Specifically, Mps1 plays an essential role in recruiting Mad1 and Mad2 to unattached kinetochores, thus mediating proper chromosome congression and accurate chromosome segregation [11-17]. Given its essential role in SAC functions, Mps1 undergoes a dynamic distribution during mitosis [10, 18]. Biochemically, Mps1 is phosphorylated on multiple residues and the phosphorylation is essential for its subcellular localization. Threonine 12 (T12) and serine 15 (S15) appear to be critical in mediating the accumulation of Mps1 on kinetochores [19]. Moreover, Mps1 inactivation is at least partly regulated by a proteasomedependent degradation process mediated by $\mathrm{APC}^{\mathrm{Cdc} 20}$ [20-22]. Timely inactivation of Mps1 is required for normal cell cycle progression as well as the termination of SAC.

Given that sumoylation plays an essential role in regulating the activity of numerous mitotic proteins, we determined whether Mps1 was also modified by sumoylation and whether the modification affected its activity during mitosis. We found that Mps1 was 
modified by both SUMO-1 and SUMO-2 during the cell cycle. Site-directed mutagenesis coupled with ectopic expression revealed that lysine residues including K71, K287, K367 and K471 were likely SUMO acceptors. Ectopic expression of SUMO-resistant Mps1 resulted in an accelerated mitotic progression. Therefore, our current study reveals a new type of post-translational mechanism that modulates Mps1's function in mitosis.

\section{RESULTS}

\section{Mps1 is modified by SUMOylation}

To study whether sumoylation plays a direct role in regulating the activity of Mps1 during the cell cycle, we transfected HEK293T cells with a plasmid construct expressing Myc-tagged Mps1, alone or along with plasmids expressing His6-SUMO-1 and FLAGUBC9. Two days after transfection, cells were treated with nocodazole for $16 \mathrm{~h}$, after which equal amounts of cell lysates prepared under the denature condition were subjected to Ni-IDA affinity pull-down analysis. Precipitated proteins were then blotted with antibodies against Myc-tag. As shown in Figure 1A, slower mobility shift bands were detected exclusively in lysates from cells expressing transfected Myc-Mps1, His6-SUMO-1 and FLAG-UBC9, strongly suggesting the presence of sumoylated Mps1. Moreover, in this transient expression system, the slow mobility shifts were observed in both asynchronized and mitotic cell lysates.

SUMO modification is a reversible process, and de-conjugation of the SUMO moiety from targeted proteins is catalyzed by sentrin-specific isopeptidases (SENPs). To further confirm the slow mobility shifts were sumoylated Mps1, HEK293T cells were transfected with plasmids expressing Myc-tagged Mps1 alone or along with constructs either expressing His6-SUMO-1 and FLAG-tagged wild-type SENP1 or His6-SUMO-1 and FLAG-tagged enzymatically defective SENP1 (SENP1-mut). Forty-eight hours post-transfection, cells were treated with nocodazole for $16 \mathrm{~h}$. Equal amounts of cell lysates prepared under the denature condition were subjected to Ni-IDA affinity pull-down analysis. Precipitated proteins were then blotted with antibodies against the Myc-tag. The slower mobility shifts of Mps 1 were completely abolished in cells expressing wild-type SENP1, but not SENP1-mut (Figure 1B, 1st and 2nd panels). Expression of FLAG-SENP1 was confirmed by blotting total lysates with antibodies against the FLAG tag and SUMO1 proteins (Figure 1B, the 3rd and 4th panels). Combined, these results strongly support the notion that ectopically expressed Mps 1 can be modified by sumoylation.

\section{Mps1 is modified by both SUMO-1 and SUMO-2}

To understand whether endogenous Mps1 is sumoylated during cell cycle progression, we took advantage of HeLa cell lines that stably expressed His6tagged SUMO-1 or His6-tagged SUMO-2. Cells were either asynchronized or treated with nocodazole or taxol for $16 \mathrm{~h}$. Equal amounts of cell lysates prepared under a denaturing condition were subjected to Ni-IDA pulldown analysis. Protein precipitates were then blotted with antibodies against Mps1 protein. As shown in Figure 2A, slow mobility bands that were immunoreactive to Mps 1 antibodies were detected in both cells expressing His6SUMO-1 and His6-SUMO-2. Moreover, the signal was enhanced when cells were treated with nocodazole or taxol. These results strongly suggest that endogenous Mps1 is sumoylated during the cell cycle.

To further confirm that endogenous Mps1 is sumoylated, HeLa cells were either asynchronized or treated with nocodazole for $16 \mathrm{~h}$. Equal amount of cell lysates prepared under a native condition were immunoprecipitated with the antibody to Mps1 and the immunoprecipitates were blotted for Mps1, SUMO-1 and SUMO-2. As shown in Figure 2B, both SUMO-1 and SUMO-2 antibodies detected a band that migrated at the same position as the one detected in the pull-down materials by the Mps1 antibody but not by the control IgG. Again, the signal was greatly enhanced when cells were treated with nocodazole. Combined, these results strongly support the notion that endogenous Mps1 is modified by both SUMO-1 and SUMO-2 during the cell cycle.

\section{Multiple lysine residues are essential for Mps1 SUMOylation}

To identify the potential lysine residues of sumoylation, we analyzed Mps1 amino acid sequences for optimal sumoylation using the criteria available at Abgent Inc. Six lysines residues (K71, K287, K336, K367, K471, $\mathrm{K} 772$ ) with the highest scores were subjected to mutagenic analysis. Mutant constructs were made that expressed Myc-tagged Mps1 with lysine residues replaced with arginines (Figure 3A). HEK293T were first transfected with indicated Mps1 constructs, along with a plasmid expressing His6-SUMO-1 for $48 \mathrm{~h}$, and then treated with nocodazole for $16 \mathrm{~h}$. Equal amounts of cell lysates prepared with a denaturing buffer were subjected to NiIDA pull-down analysis. The precipitates were blotted with the antibody against the Myc-tag. As shown in Figure 3A, wild-type Myc-Mps 1 and its mutant constructs were expressed at a comparable level (Figure 3B and 3C, input), suggesting that sumoylation may not affect the protein stability. Importantly, an extra slower mobility 
A

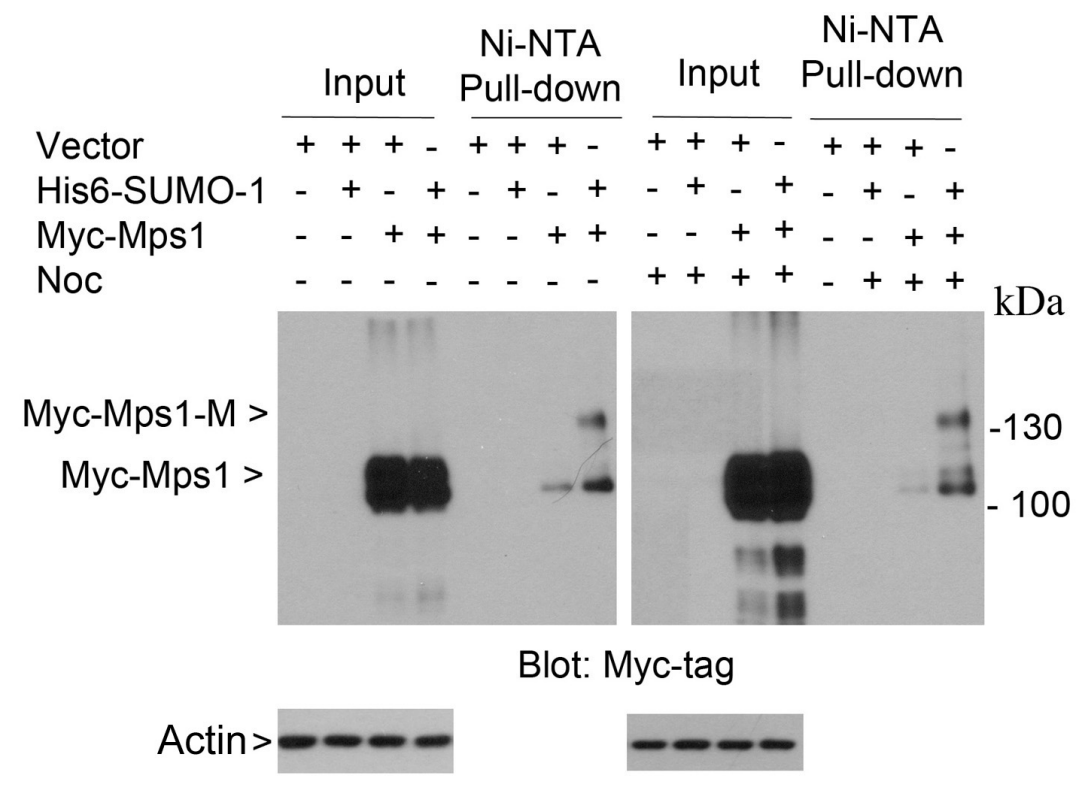

B
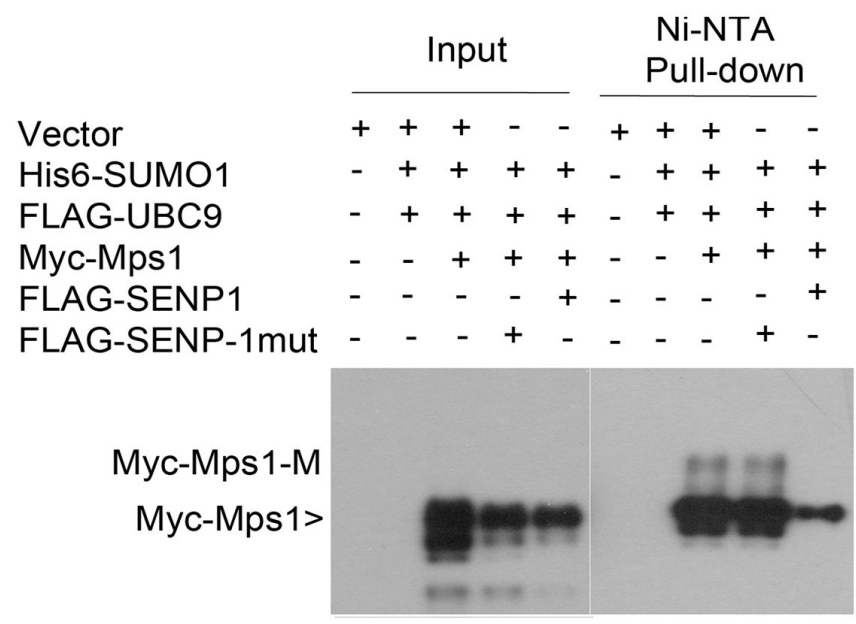

Blot : Myc-Tag
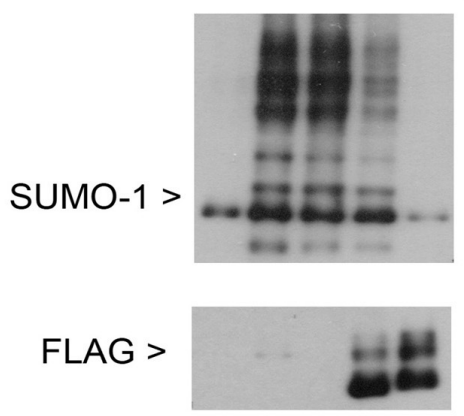

Actin >

Figure 1: Sumoylation of ectopically expressed Mps1. (A) HeLa cells were transfected with plasmids as indicated for $48 \mathrm{~h}$ followed by treatment with nocodazole for $16 \mathrm{~h}$. Equal amounts of total cell lysates prepared in the $8 \mathrm{M}$ urea lysis buffer were subject to $\mathrm{Ni}$-IDA pull-down analysis. Protein precipitates were then blotted for Myc-tag. Total lysates were also blotted with the antibody to $\beta$-actin. Myc-Mps1-M indicates the modified form of Myc-Mps1. (B) HeLa cells were transfected with various plasmids as indicated. Ni-IDA pulldown assay was performed as described in A. Protein precipitates were blotted for Myc-tag. Total lysates were also blotted for SUMO-1, FLAG-tag and $\beta$-actin. 
A

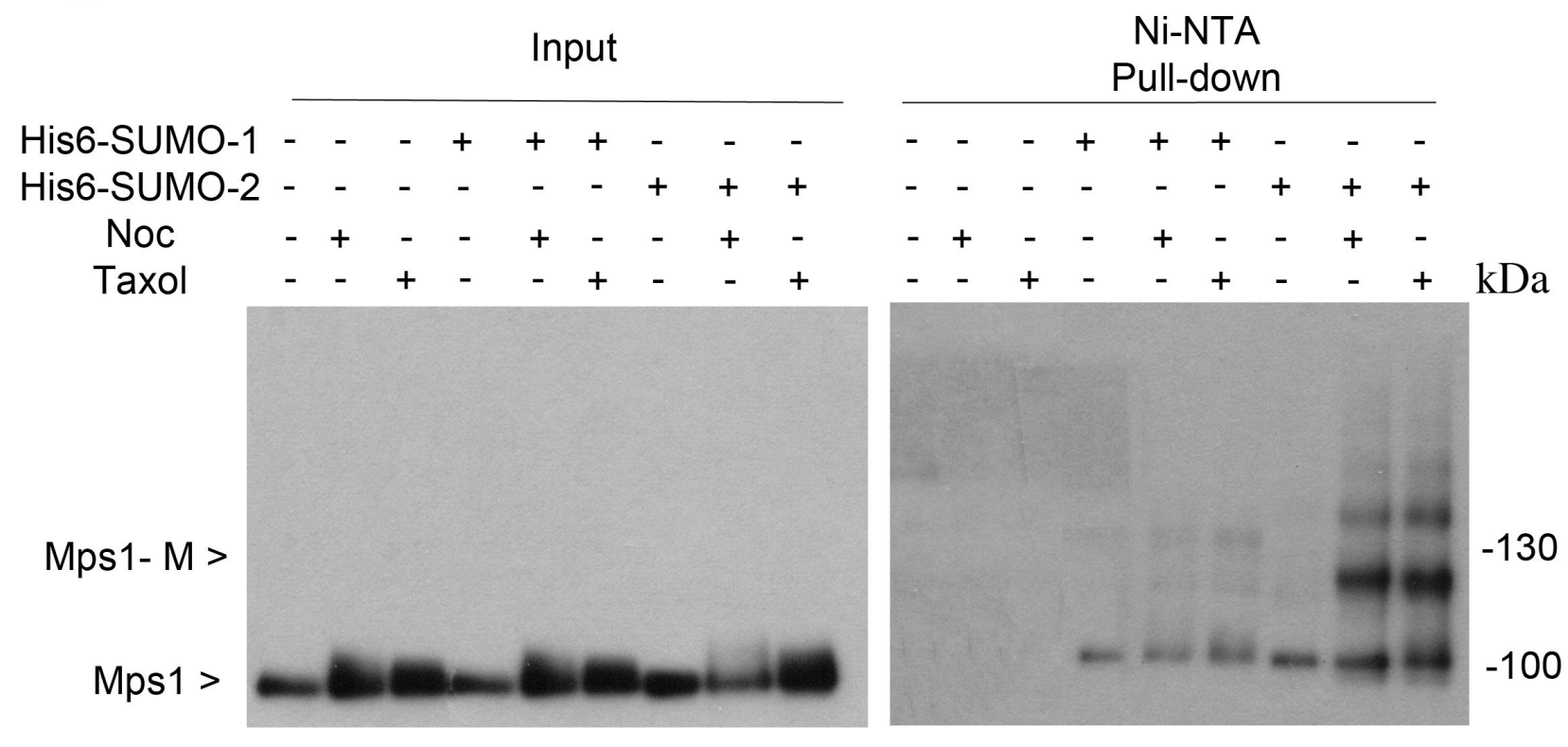

Blot: Mps1

Actin >

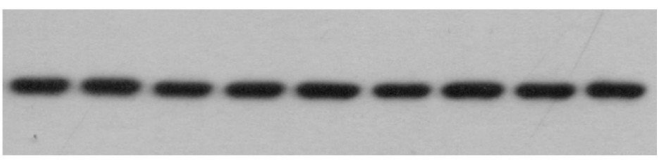

B

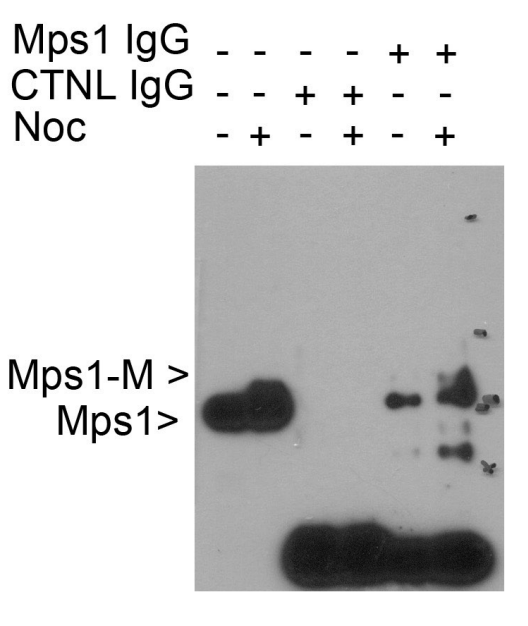

Blot: Mps1
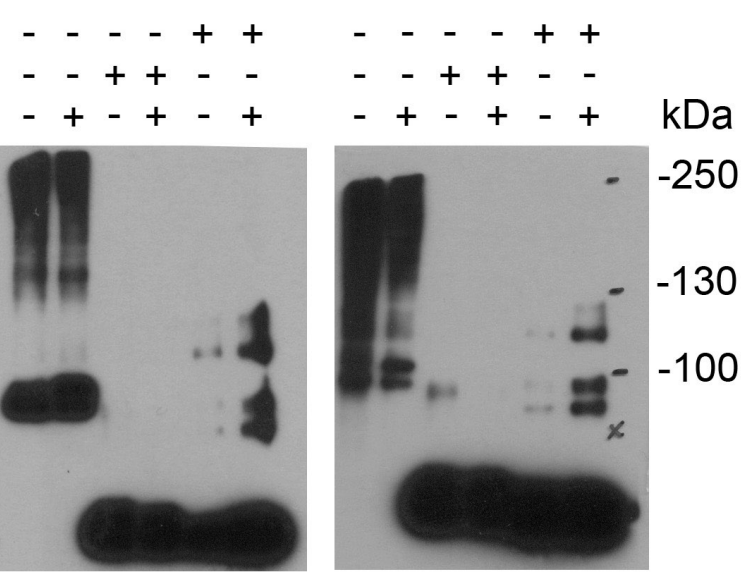

Blot: SUMO-1
Blot: SUMO-2

Figure 2: Sumoylation of endogenous Mps1. (A) HeLa cells stably expressing His6-SUMO-1 or His6-SUMO-2 proteins were treated with nocodazole or taxol for $16 \mathrm{~h}$, after which cells were lysed and subject to Ni-IDA pull-down assay under denature conditions. Protein precipitates, along with lysate inputs were blotted for Mps 1 and $\beta$-actin. (B) HeLa cells were treated with nocodazole for 16 h, after which cells were lysed and equal amounts of cell lysates prepared in a native condition were immunoprecipitated with Mps1 antibody or a control mouse IgG. Immunoprecipitates were blotted for Mps1, SUMO-1, and SUMO-2. Mps1-M indicates the SUMO modified Mps1. 
A

$\begin{array}{lllll}\text { K71 } & \text { K287 } & \text { K336 K367 } & \text { K471 } & \text { K771 }\end{array}$

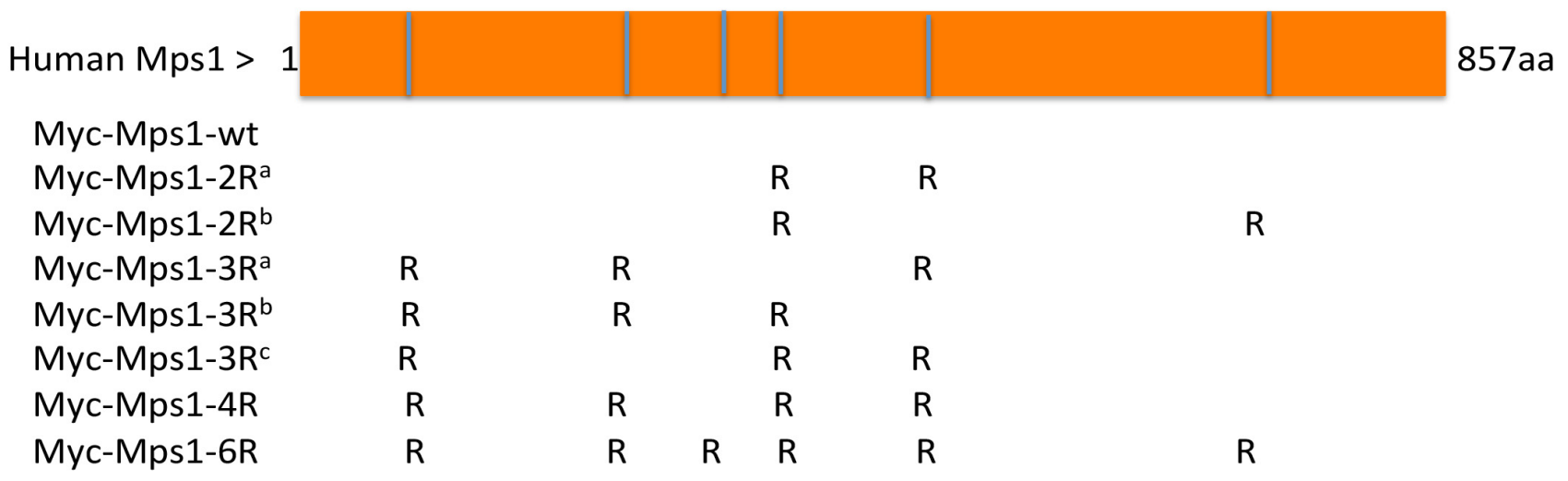

B
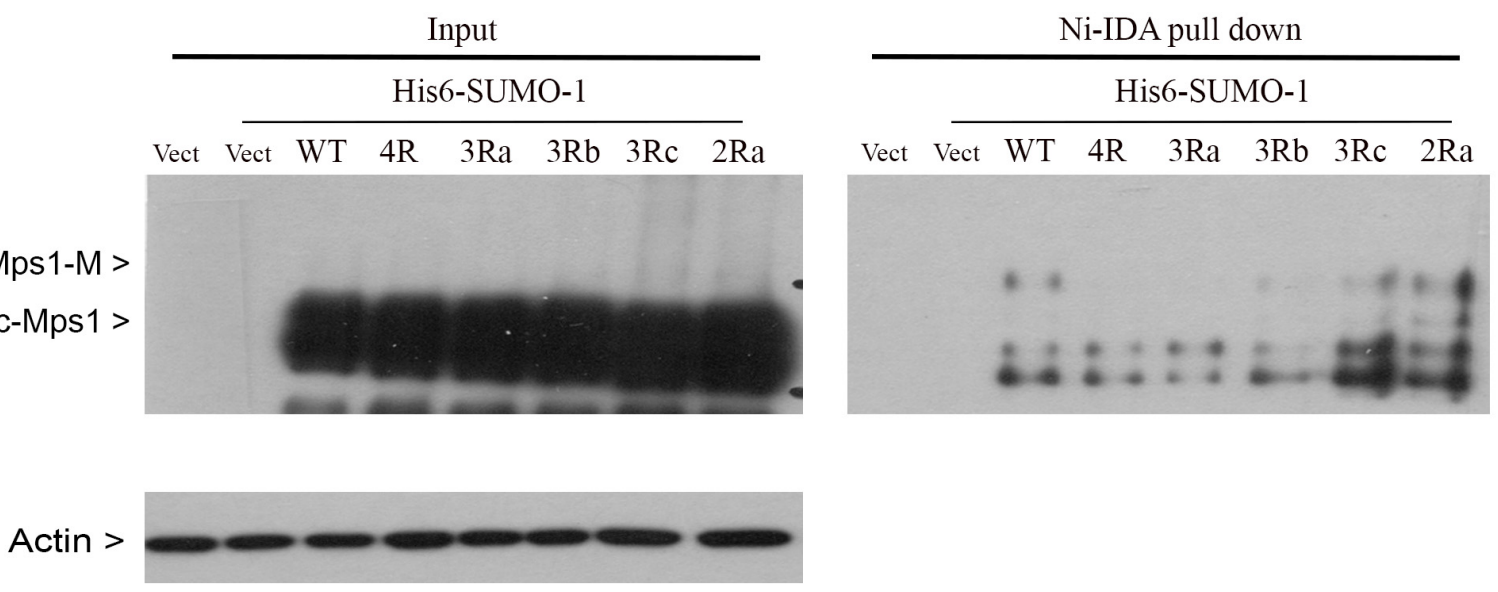

C

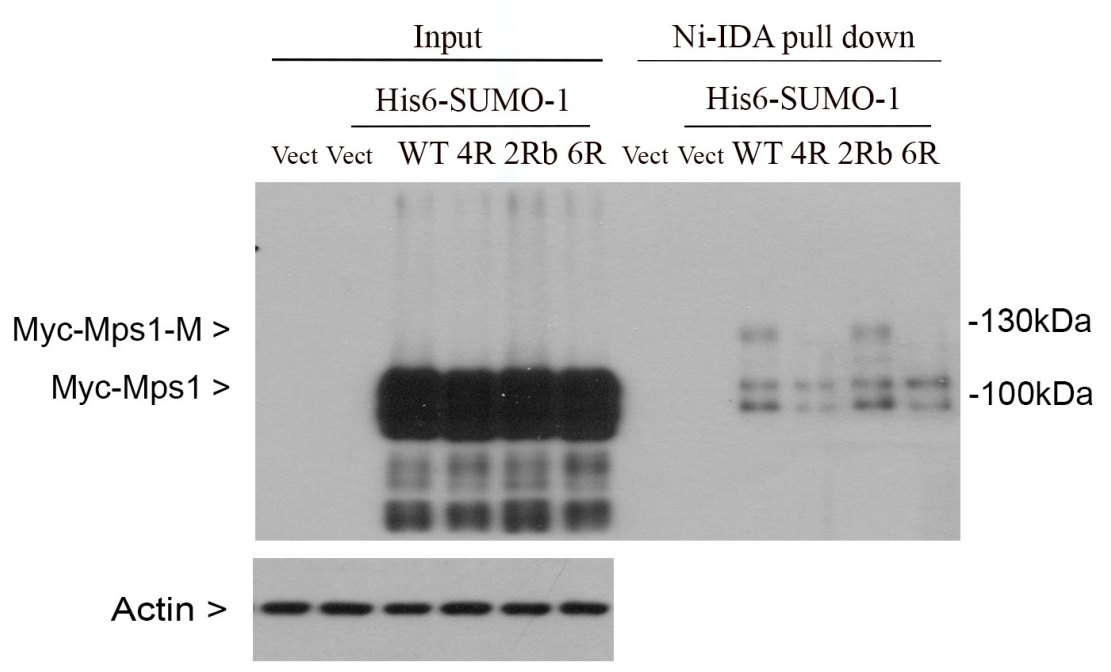

Figure 3: Identification of lysine residues essential for Mps1 sumoylation. (A) Schematic presentation of human Mps1 wildtype and various mutants used in this study. (B and C) HEK293T cells were transfected with empty vector (vect) or various Mps1 plasmids together with constructs expressing His6-SUMO-1 protein for $48 \mathrm{~h}$ followed by treatment with nocodazole for $16 \mathrm{~h}$. Total cell lysates prepared under denature conditions were subject to Ni-IDA pull-down assay. Protein precipitates, along with lytsate inputs, were blotted for the Myc-tag and $\beta$-actin. 
band that was immunoreactive to the Myc antibody was detected in cells expressing wild-type or mutant with K336R and K471R but not mutant with all 6 lysine residues replaced with arginies or mutants with 4 lysine residues mutated K71, K287, K367 and K471 (referred as 4R mutants hereafter). Combined, these results indicate that lysines K71, K287, K367 and K471 are essential for Mps1 sumoylation.

\section{SUMOylation partially affects Mps1 subcellular localization during mitosis}

Sumoylation plays a role in regulating the subcellular localization of target proteins [25-27]. To test whether this is the case for Mps1, HeLa cells were transfected with GFP-tagged wild-type Mps1 (GFPMps1-wt) or its SUMO-resistant 4R mutants (GFP-Mps1mut) for $48 \mathrm{~h}$. Cells were then collected for subcellular fractionation, after which equal amounts of cell lysates were blotted with the antibody against GFP. As shown in Figure 4A (left panel), we did not see apparent subcellular localization difference between GFP-Mps1-wt and its SUMO-resistant counterpart although a relatively lower level of GFP signal was detected in cells transfected with GFP-Mps1-Mut. Next we treated the transfected cells with nocodazole for $16 \mathrm{~h}$. After fractionation, GFP-tag signals were detected by Western blotting. Interestingly, compared with GFP-Mps1-wt, higher levels of GFP-Mps1-mut were detected in the fraction of loose chromatin-binding proteins (Figure 4A, right panel).

Kinetochore localization is required for the mitotic function of Mps1 [28, 29]. To test whether sumoylation regulates Mps1 kinetochore localization, HeLa cells were transfected with a plasmid construct expressing Myc-tagged wild-type Mps1 (Myc-Mps1-wt) or its SUMO-resistant 4R mutant (Myc-Mps1-Mut) for $48 \mathrm{~h}$, followed by treatment with $40 \mathrm{ng} / \mathrm{mL}$ nocodazole for $4 \mathrm{~h}$. Mitotic chromosome spreads were prepared, and kinetochore localization of Myc-Mps1 was detected by immunofluorescent staining with antibodies against the Myc-tag. As shown in Figure 4B, both Myc-Mps1-wt and the mutant counterpart Myc-Mps1-mut showed clear kinetochore localization; however, the signal intensity was stronger in cells expressing Myc-Mps1-mut (Figure 4C).

\section{Mps1 SUMOylation regulates mitotic progression}

We next determine whether sumoylation-resistant mutant of Mps1 would affect mitotic progression. HeLa cells were transfected with a plasmid construct expressing Myc-Mps1-wt or Myc-Mps1-mut for $48 \mathrm{~h}$, followed by treatment with nocodazole for $14 \mathrm{~h}$. Mitotic round-up cells were collected by physical shake-off and then subjected to mitotic release. Cells were collected at various time points after release and equal amounts of lysates were subjected to Western blot analysis. As shown in Figure 5A, Mps1 levels peaked at prometaphase/metaphase stages in control cells, after which it gradually decreased correlated with the decline of both cyclin B1 and phosphorylated histone H3 (p-H3S10) signals, indicating that Mps1 may also mediate the mitotic exit. Interestingly, compared to cells expressing Myc-Mps1-wt, disappearance of cyclin B1 and $\mathrm{p}-\mathrm{H} 3^{\mathrm{s} 10}$ accelerated in cells expressing Myc-Mps1mut (Figure 5B, 5C and 5D), suggesting that sumoylation of Mps1 may be involved in mitotic timing. We next transfected HeLa cells with plasmids expressing GFPMps1-wt or GFP-Mps1-mut together with siRNAs specific to endogenous Mps1. Twenty-four hours after transfection, cells with GFP signals were subject to analysis using timelapse confocal microscopy. As shown in Figure 5E and $5 \mathrm{~F}$, the mitotic time of cells expressing GFP-Mps1-mut was shorter than that of cells expressing GFP-Mps1-wt $(37.50 \pm 6.21 \mathrm{~min}$. vs. $43.50 \pm 7.69 \mathrm{~min})$. These results further support the notion that sumoylation plays a role in regulating the mitotic function of Mps1. To confirm the specific knockdown of endogenous of Mps1 by siRNAs, HeLa cells were transfected with either control luciferase siRNAs or siRNAs targeting Mps 1 3'-UTR region together with plasmids expressing Myc-Mps1 for 24 hours, followed by the nocodazole treatment for 16 hours. Equal amount of cell lysates was blotted for antibodies against Mps1 and actin. As shown in Figure 5G, endogenous Mps1 was greatly reduced in cells transfected with Mps1specific siRNAs, and the expression of Myc-Mps1 was not affected. Notably, the slightly slower mobility shift in cells expressing Myc-Mps1 was caused by the Myc tag.

\section{SUMOylation regulates the interaction of Mps1 with BubR1}

Mps1 is known to be the up-stream regulator of SAC [30]. We then asked whether sumoylation would affect the interaction between Mps1 and other SAC components. HeLa cells were either asynchronized or treated with nocodazole for $16 \mathrm{~h}$. Equal amounts of total cell lysates were subject to immunoprecipitation with antibodies against BubR1 or Mps1, and then blotted for Mps1. As shown in Figure 6A, Mps1 was specifically precipitated by Mps1 antibody but not by the control IgG. Notably, Mps1 was also efficiently precipitated by BubR1, but not a control, antibody, strongly suggesting the physical interaction between Mps1 and BubR1. We next transfected HeLa cells for $48 \mathrm{~h}$ with plasmids expressing Myc-Mps1-wt, Myc-Mps1-mut, or the control plasmid. After treatment with nocodazole for another $16 \mathrm{~h}$, equal amounts of cell lysates were immunoprecipitated with the antibodies against MYC tag and BubR1. As shown in Figure 6B, ectopically expressed Mps1 was efficiently expressed and precipitated by the anti-Myc antibody. BubR1 was also precipitated by the Myc antibody. Significantly, a greater amount of BubR1 was precipitated 
A

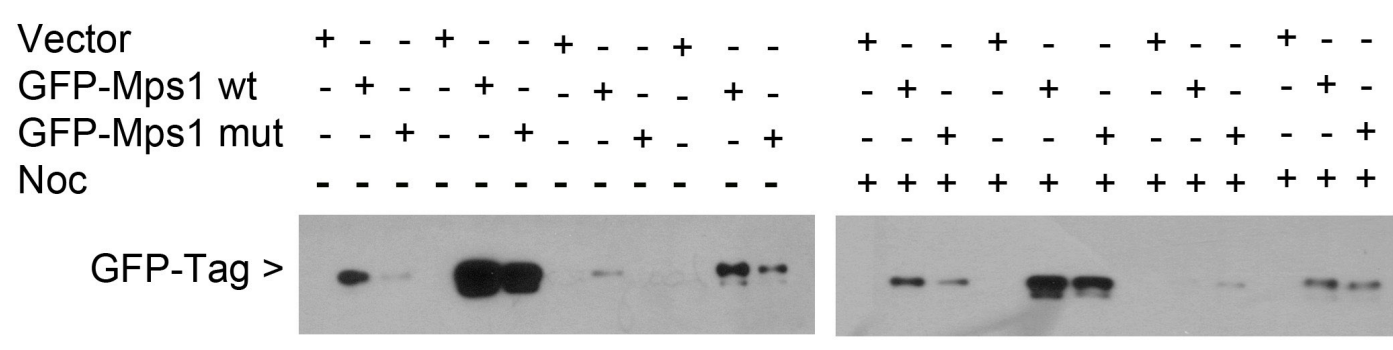

Tubulin >
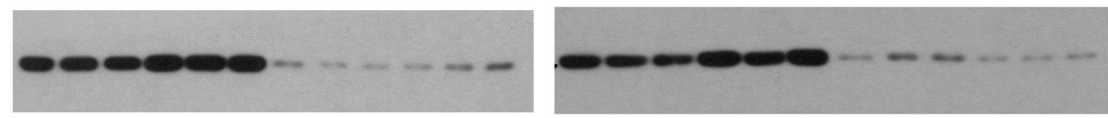

PARP-1 >
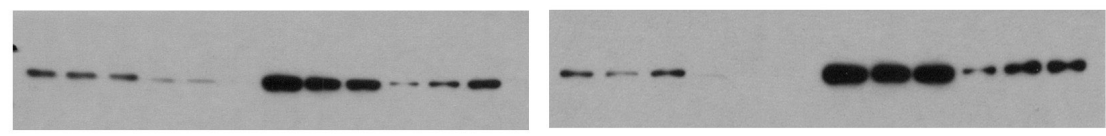

Actin >
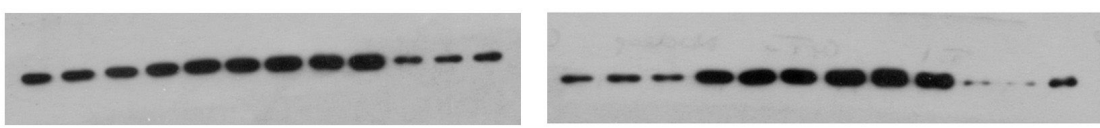

B
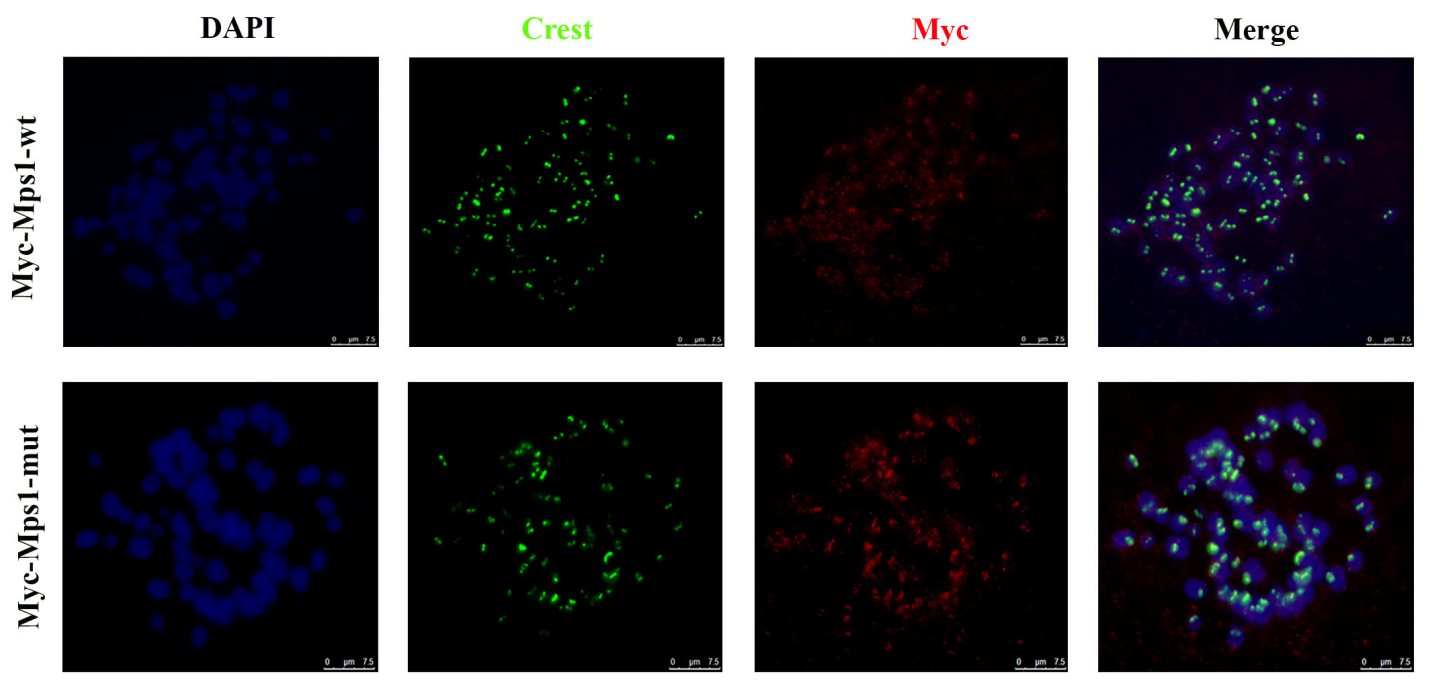

C

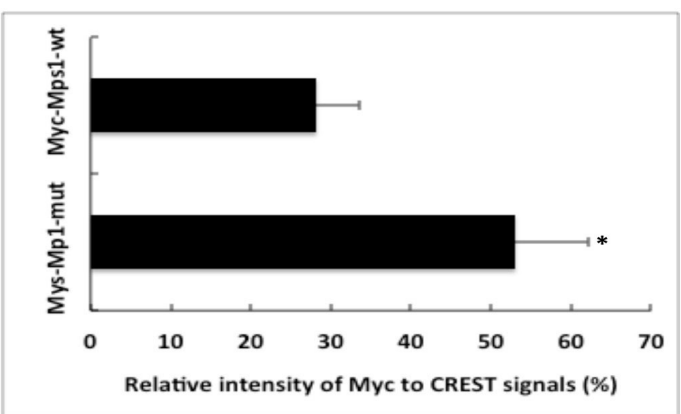

Figure 4: Sumoylation does not significantly affect Mps1 subcellular localization. (A) HeLa cells were transfected with indicated plasmids for $48 \mathrm{~h}$ followed by treatment with nocodazole for $16 \mathrm{~h}$. Cells were then collected for fractionation to isolate cytoplasmic, soluble nuclear, and chromatin binding proteins. Fractionated proteins were then blotted for GFP, $\alpha$-tubulin, PARP-1 and $\beta$-actin. (B) HeLa cells were transfected with plasmids expressing Myc-Mps1-wt or Myc-Mps1-mut for $48 \mathrm{~h}$ followed by treatment with nocodazole for $4 \mathrm{~h}$ prior to chromosome spread analysis. Chromosome spreads were stained overnight with antibodies against Myc-tag and CREST. DNA was counter-stained with DAPI. Representative images show the kinetochore localization of Myc-Mps1. (C) The fluorescent intensity of MycMps1 from (B) was analyzed using ImageJ software and normalized to the CREST signal (Myc-Mps1-wt: $n=11$, Myc-Mps1-mut: $n=10$ ). 
A
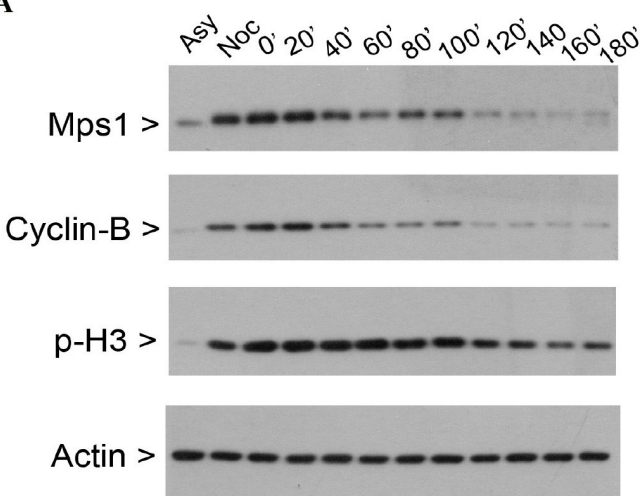

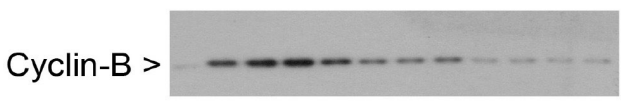

B

Myc-Tag >

Cyclin-B >

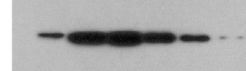

$\mathrm{pH} 3>$

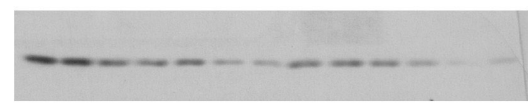

Actin $>$

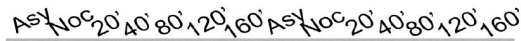

Nocodazole Release

Myc-Mps1 wt Myc-Mps1 mut 1
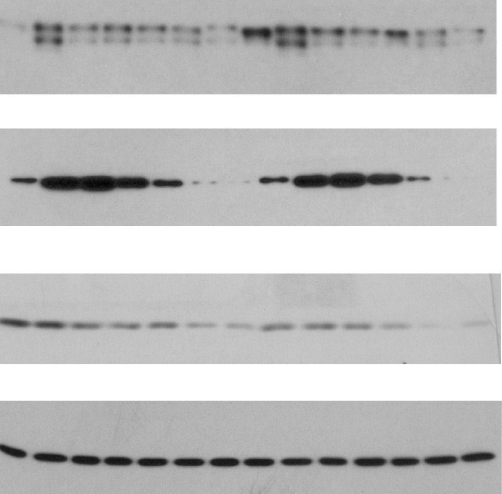

G

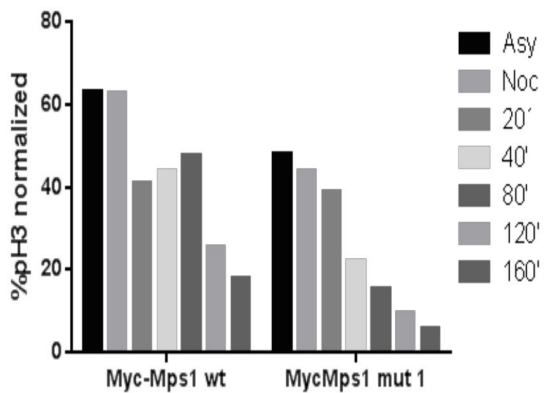

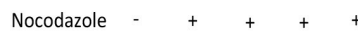
Luc-siRNA Mps1-siRNA Myc-Mps1

Mps1>

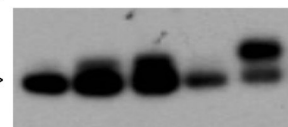

Actin>

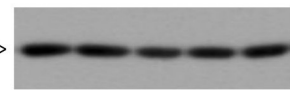

E
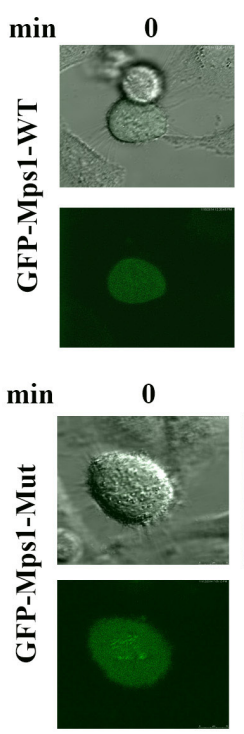

6

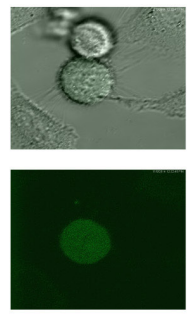

6

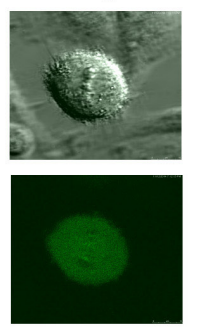

12
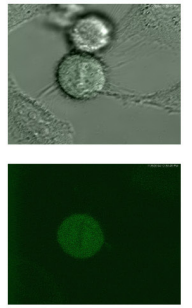

12

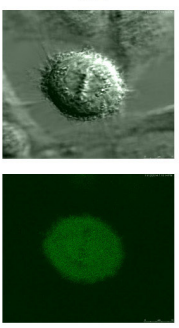

18
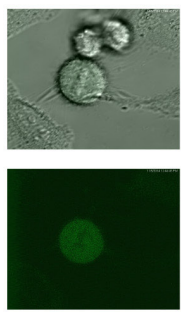

18

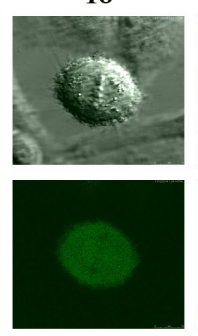

24
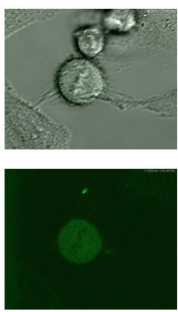

24

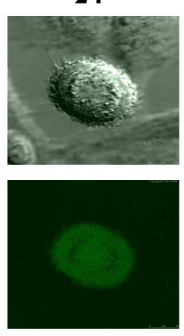

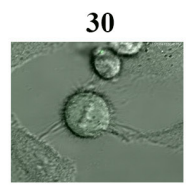
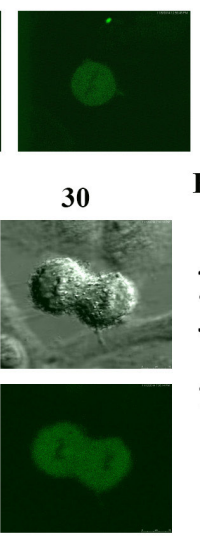
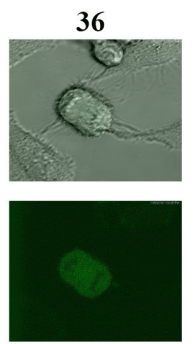

F 60

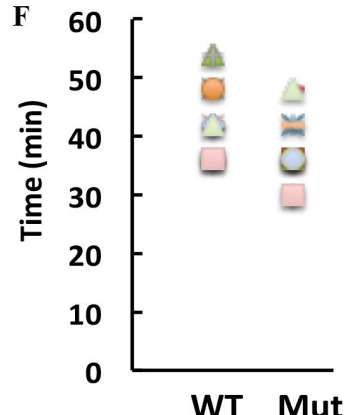

Figure 5: Sumoylation of Mps1 affects mitotic progression. (A) HeLa cells were treated with nocodazole for 14 h. Round-up cells were harvested by physical shake-off and subject to mitotic release for various times. Equal amounts of cell lysates were blotted for Mps1, cyclin B1, phospho-histone H3S10 (p-H3) and $\beta$-actin. (B) HeLa cells were transfected with plasmids expressing Myc-Mps1-wt or Myc-Mps1-mut for $48 \mathrm{~h}$ followed by treatment with nocodazole for $14 \mathrm{~h}$. Mitotic cells were then release into the cell cycle. Equal amounts of lysates were then blotted for indicated antibodies. (C and D) Quantitation of the degradation rates of cyclin B (C) and pH3 from (B). (E) HeLa cells were transfected with siRNAs specific to Mps1 and plasmids expressing GFP-Mps1-wt or GFP-Mps1-mut for 24 h followed by time-lapse confocal microscopy. Representative images from are shown. (F). Summary of data obtained from experiments shown in (E) to show mitotic duration of cells expressing either GFP-WT $(n=8)$ and GFP-5R $(n=8)$. (G) HeLa cells were transfected with either control luciferase siRNAs or siRNAs targeting Mps 1 3'-UTR region together with plasmids expressing Myc-Mps1 for 24 hours, followed by the nocodazole treatment for 16 hours. Equal amount of cell lysates prepared in $8 \mathrm{M}$ urea was blotted for Mps1 and actin. 
from cells expressing Myc-Mps1-mut than that of the cells expressing Myc-Mps1-wt, strongly suggesting that sumoylation may regulate the interaction of Mps1 with BubR1.

\section{DISCUSSION}

In this study, we show evidence that Mps1 is modified by both SUMO-1 and SUMO-2 during the cell cycle and that this post-translational modification occurs on multiple lysine residues including K71, K287, K367 and K471. Our study also shows that sumoylation may negatively affect Mps1's kinetochore localization during mitosis. Sumoylation appears to decelerate mitotic progression as mitotic cells expressing sumoylationresistant mutant of Mps1 exit from mitosis faster than those expressing wild-type Mps1. Moreover, sumoylation regulates the physical interaction of Mps1 with BubR1 during mitosis, suggesting its possible role in SAC regulation.

\section{A}

$\begin{array}{lllllllllll}\text { CNTL IgG } & - & - & + & + & - & - & + & + & - & - \\ \text { BubR1 lgG } & - & - & - & - & + & + & - & - & - & - \\ \text { Mps1 IgG } & - & - & - & - & - & - & - & - & + & + \\ \text { Nocodazole } & - & + & + & - & + & - & + & - & + & -\end{array}$

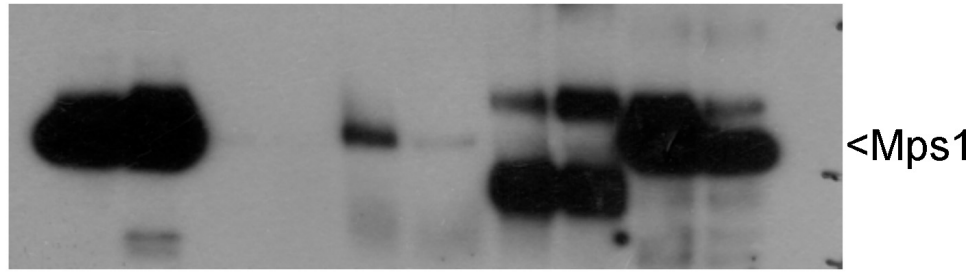

Blot:: Mps1

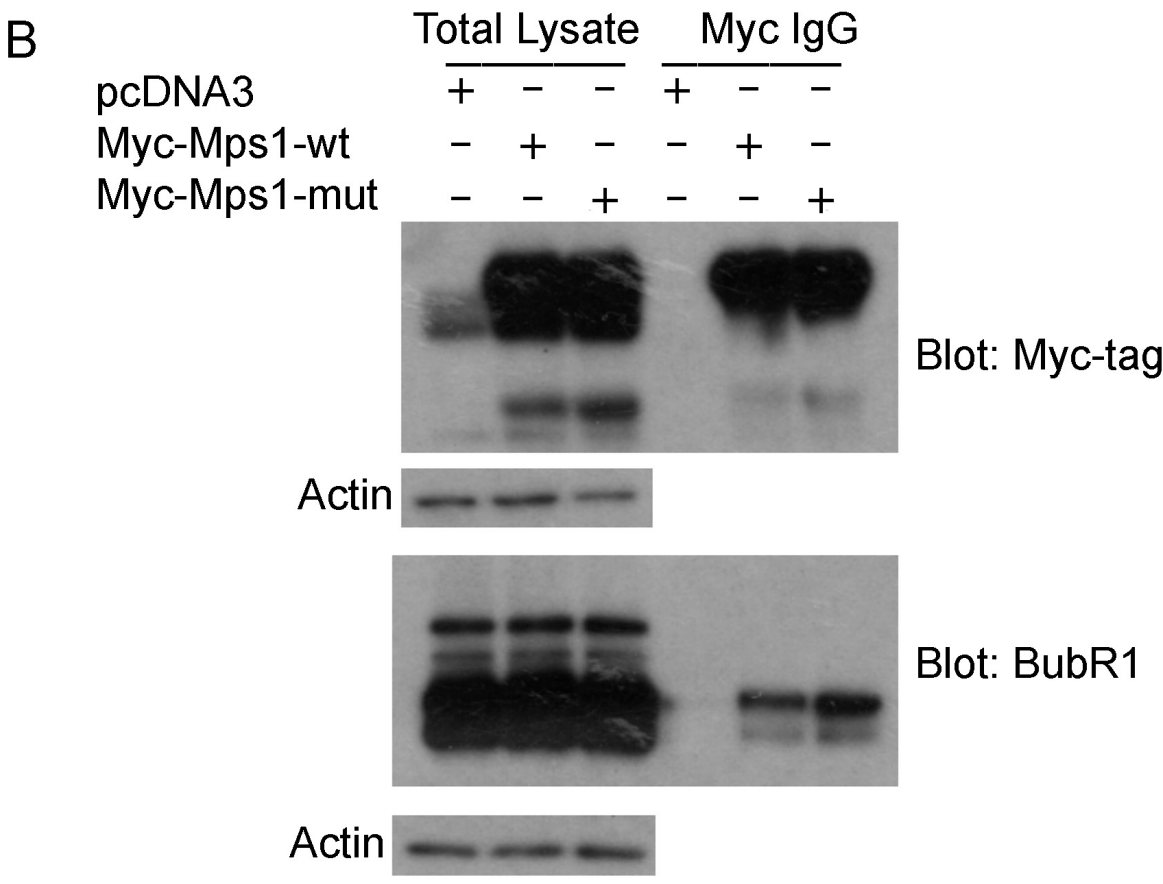

Figure 6: Mps1 sumoylation regulates its interaction with mitotic checkpoint protein BubR1. (A) HeLa cells were treated with or without nocodazole for $16 \mathrm{~h}$ after which cells were lysed in a native condition and immunoprecipitated with antibodies to BubR1 or Mps1. Control IgGs were also used as controls. Protein precipitates were then blotted for Mps1. (B) HeLa cells were transfected with Myc-Mps1-wt, Myc-Mps1-mut, or pcDNA3 vector for $48 \mathrm{~h}$ followed by treatment with nocodazole for $16 \mathrm{~h}$. Total cell lysates prepared in a native condition were immunoprecipitated with the antibody to Myc. Protein precipitates were then blotted for Myc-tag and BubR1. Total lysates were also blotted for $\beta$-actin. 
The N-terminus of Mps1 is important for its nuclear translocation and kinetochore localization [9, 21, 31-35]. As all four lysine residues identified for SUMO modification reside in the $\mathrm{N}$-terminal region of $\mathrm{Mps} 1$ we speculated that this modification may play a significant role in its subcellular localization. Intriguingly, protein fractionation of asynchronized cells did not reveal a major difference between cells expressing GFP-Mps1-wt and its SUMO-resistant mutant counterpart. On the other hand, Myc-Mps1-mut was enriched at the kinetochores of mitotic chromosomes more than it did with wildtype counterpart (Figure 4B). Moreover, when SAC was activated by nocodazole, enhanced chromatin binding was observed in cells expressing SUMO-resistant mutants (Figure 4A), suggesting that sumoylation may play a role in modulating kinetochore localization of Mps1. Consistent with this notion, many studies have shown that sumoylation is an important mechanism by which mitotic components assemble a functional kinetochore during mitosis [36-38].

As an Mps1 target, BubR1 plays critical roles in regulating SAC and mitotic timing. Physical interaction between Mps1 and BubR1 is observed in Drosophila [39]. However, it has not observed in mammalian cells [40]. In our current study, we demonstrated that endogenous Mps1 was precipitated by a BubR1-specific antibody, indicating their interaction. Endogenous BubR1 was also precipitated by the Myc-tag antibody in cells ectopically expressing Myc-Mps1. Combined, these results support the conclusion that Mps1 physically interacts with BubR1 in vivo in mammalian cells. Although the molecular mechanism remains unclear, sumoylation appears to negatively regulate the association of $\mathrm{Mps} 1$ with BubR1 in mitosis because increased level of BubR1 was pulled-down by Myc-Mps1-mut than that by MycMps1-wt. In other words, the interaction between Mps1 and BubR1 may be fine-tuned through post-translational SUMO modification. Moreover, these observations also suggest that BubR1 kinetochore localization may be mediated, at least partially, through Mps1 sumoylation. Supporting this, it has been shown that although Mps1 is not required for the recruitment of BubR1 to unattached kinetochores [9] its absence increases the rate of BubR1 exchange on the kinetochores [39].

Mps1 primarily functions as a mitotic kinase [31, 32]. Although identified four lysine residues essential for sumoylation reside in the N-terminus, we are not sure whether sumoylation will change the overall protein structure, consequently influencing the kinase activity towards its targets including BubR1. Given that inhibition of Mps1 kinase activity leads to enhanced kinetochore localization $[11,15,41]$, we speculate that sumoylation will positively influence Mps1 kinase activity as cells expressing SUMO-resistant Mps1 display an increase level of kinetochore localization (Figure 4B).

Sumoylation regulates mitotic progression via monitoring chromosome congression, chromosome alignment, and anaphase onset [38, 42]. Depletion of Mps1 by siRNA causes dysfunction of kinetochores along with premature mitotic exit. In our study, cells expressing GFP-Mps1-mut coupled with depletion of endogenous Mps1 exhibited a shortened mitotic duration than those expressing GFP-Mps1-wt (Figure 5). Intriguingly, we did not observe increased premature chromosome separation, suggesting partial rescuing of premature chromosome segregation. These results also suggest that SUMOdeficient mutants fail to fully rescue impaired SAC function due to Mps1 depletion. Since cells that are lack of functional Mps1 become aneuploid and subsequently die $[43,44]$ it will be of great interest to know whether daughter cells that express GFP-Mps1-mut display increased chromosome missegregation.

It has also been shown that Mps1 plays a role in regulating centrosome function [10, 45-48]. In our current study, we did not see clear centrosome localization of ectopically expressed GFP-Mps1 or GFP-Mps1-mut in interphase cells, which is likely due to the fact that only a small proportion of Mps1 localizes to the centrosome. Interestingly, when cells expressing GFP-Mps1-mut or GFP-Mps1-wt were treated with nocodazole followed by the release into the cell cycle, a higher proportion of mutant-expressing cells displayed aberrant centrosome structures (e.g., tri-centrosomes) than those expressing GFP-Mps1-wt (data not shown). Thus, sumoylation may mediate Mps1's centrosomal function during mitosis.

\section{MATERIALS AND METHODS}

\section{Cell culture and transfection}

HeLa and HEK293T cell lines were obtained from the American Type Culture Collection. HeLa cells stably expressing His6-SUMO-1 or His6-SUMO-2 were kindly provided by R.T. Hay [23]. Cells were cultured in DMEM supplemented with $10 \%$ fetal bovine serum (FBS, Invitrogen) and antibiotics $(100 \mu \mathrm{g} / \mathrm{ml}$ of penicillin and $50 \mu \mathrm{g} / \mathrm{ml}$ of streptomycin sulfate, Invitrogen) at $37^{\circ} \mathrm{C}$ under $5 \% \mathrm{CO}_{2}$. The stable cell lines were maintained under $2 \mathrm{mM}$ of puromycin (Sigma-aldrich).

Transfection of plasmids or siRNAs was achieved with either Fugene HD (Roche Diagnostic) or Lipofectamine 2000 (Invitrogen) following the manufacturer's instructions. Mitotic shake-off cells were obtained from gentle tapping of cells treated with nocodazole or taxol $(50 \mathrm{ng} / \mathrm{ml})$ (Sigma-Aldrich) for $14 \mathrm{~h}$. Both types of shake-off cells were used for mitotic release in the presence or absence of nocodazole as specified.

\section{Antibodies}

Rabbit polyclonal antibodies for BubR1 were developed in the laboratory. Antibodies for Myc-Tag, p-H3S10, Cyclin B1, and $\beta$-actin were purchased from Cell Signaling Technology Inc. Mouse monoclonal antibody 
for Mps1 was purchased from Invitrogen. GFP antibodies were purchased from Santa Cruz Biotechnology. Mouse anti-FLAG and anti- $\alpha$-Tubulin were purchased from Sigma Aldrich. Mouse anti-SUMO-1 and SUMO-2/3 antibodies were kindly provided by Dr. Michael J. Matunis (John Hopkins University). Human IgGs (CREST) against centromere proteins were purchased from Antibodies Incorporated (Davis, CA).

\section{Plasmids, mutagenesis, and siRNAs}

Various Mps1 mutants with lysine residues (K71, K287, K336, K367, K471 and/or K772) replaced with arginines (R) were generated using the QuickChange Lightning Multi Site-directed Mutagenesis kit (Stratagene). Individual mutations were confirmed by DNA sequencing (Seqwright). His6-SUMO-1 plasmid was purchased from Addgene. FLAG-SENP-1 and its mutant expression plasmids were kindly provided by $\mathrm{J}$. Cheng [24]. UBC9 cDNA was subcloned into a FLAGexpression vector. Synthetic siRNA specific to the Mps1 gene 3 '-untranslated region (5'-UUG-CUA-UCC-ACCCAC-UAU-UUU-3'), as well as the control siRNA, were purchased from Dharmacon RNAi Technology.

\section{Western blot}

SDS-PAGE was carried out using the mini-gel system from Bio-Rad. Proteins were transferred to PVDF (Polyvinylidene fluoride) membranes. After blocking with TBST buffer (50 mM Tris, $150 \mathrm{mM} \mathrm{NaCl}, 0.05 \%$ Tween 20 ) containing 5\% nonfat dry milk for $1 \mathrm{~h}$, the membranes were incubated overnight with primary antibodies, followed by incubation with horse-radish peroxidaseconjugated secondary antibodies (Cell Signaling Technology) for $1 \mathrm{~h}$ at room temperature. After thorough washing with TBST buffer, signals were developed with an enhanced chemiluminescent system (Pierce).

\section{Immunoprecipitation and pull-down assays}

For immunoprecipitation, cells were lysed in a lysis buffer (20 mM Tris, pH 7.5, $150 \mathrm{mM} \mathrm{NaCl,} \mathrm{1 \%} \mathrm{Triton,}$ $2 \mathrm{mM}$ sodium pyrophosphate and $1 \mathrm{mM}$ EDTA, $1 \mathrm{mM}$ $\mathrm{NaF}, 1 \mathrm{mM}$ sodium orthovanadate, $500 \mathrm{mM}$ PMSF, $2 \mathrm{mM}$ pepstatin A, 10 units/ml aprotinin, $20 \mathrm{mM} \mathrm{NEM}$ ) and cleared by centrifugation. $1 \mu \mathrm{g}$ of antibody and $25 \mu \mathrm{l}$ of protein G-agarose resin (50/50, Millipore) were then added to $2 \mathrm{mg}$ cell lysates and incubated at $4^{\circ} \mathrm{C}$ overnight followed by five washing with the lysis buffer. Proteins bound to resin were eluted with SDS sample buffer and then subject to analysis by SDS-PAGE followed by Western blot with appropriate antibodies.

For pull-down assays under denaturing conditions, cells were lysed in a lysis buffer (8 M urea, $50 \mathrm{mM}$ $\mathrm{Na}_{2} \mathrm{HPO}_{4} / \mathrm{NaH}_{2} \mathrm{PO}_{4}$ (pH 7.4), $300 \mathrm{mM} \mathrm{NaCl}, 0.1 \%$
Triton X-100) containing $20 \mathrm{mM}$ imidazole. Ni-IDA Superflow Resin (Clontech) was added to the cell lysates and incubated with gentle agitation at room temperature for $2 \mathrm{~h}$. The resin was then washed 3 times at room temperature with the lysis buffer containing $40 \mathrm{mM}$ imidazole. After the last wash, His6-tagged proteins were eluted in the lysis buffer containing $300 \mathrm{mM}$ imidazole and blotted for Mps1, the MYC-tag, SUMO-1, or SUMO-2.

\section{Protein fractionation}

Cells washed twice with cold PBS were lysed in buffer 1 (10 mM Tris- $\mathrm{HCl} \mathrm{pH} 7.9,10 \mathrm{mM} \mathrm{KCl,} 1.5 \mathrm{mM}$ $\mathrm{MgCl}_{2}, 1 \mathrm{mM}$ DTT) and left on ice for $10 \mathrm{~min}$. After centrifugation $(2000 \times \mathrm{g} \times 10 \mathrm{~min})$ the supernatant (the cytoplasmic extract) were collected and the pellet fraction was re-suspendend in buffer $2(20 \mathrm{mM}$ Tris- $\mathrm{HCl} \mathrm{pH} 7.9$, $1.2 \mathrm{mM} \mathrm{KCl}, 1.5 \mathrm{mM} \mathrm{MgCl}, 25 \%$ (w/v) glycerol, $0.2 \mathrm{mM}$ EDTA) followed by gentle agitation for $1 \mathrm{~h}$ at $4{ }^{\circ} \mathrm{C}$. The supernatants (the nucleoplasmic/loose chromatin binding fraction) were harvested after centrifugation (16,000 $\mathrm{g} \times 15 \mathrm{~min}$ ). The pellets (chromatin binding) were resuspended in a lysis buffer ( $8 \mathrm{M}$ urea, $50 \mathrm{mM} \mathrm{Na} \mathrm{HPO}_{4} /$ $\mathrm{NaH}_{2} \mathrm{PO}_{4}$ (pH 7.4), $300 \mathrm{mM} \mathrm{NaCl}, 0.1 \%$ Triton X-100).

\section{Mitotic chromosome spreads and fluorescence microscopy}

To obtain mitotic chromosome spreads, cells transfected with various expression constructs were treated with $40 \mathrm{ng} / \mathrm{mL}$ nocodazole for $4 \mathrm{~h}$ and then collected after Trypsin-EDTA treatment and incubated in $40 \% \mathrm{PBS}$ at $37^{\circ} \mathrm{C}$ for $15 \mathrm{~min}$. Cells were resuspended in a fixative solution (glacial acetic acid/methanol in a ratio of 1:3) prior to spreading onto microscope slides (Fisher Scientific). Chromosome spreads were incubated overnight with antibodies to Myc-tag and CREST. After incubation with Alex Fluor 488-conjugated goat anti-mouse IgG and Alex Fluor 555-conjugated goat anti-human IgG, cells were counterstained with 4, 6-diamidino-2-phenylindole (DAPI, Molecular Probe, Eugene, OR). Chromosomal images were captured with a Leica TCS SP5 confocal microscope or a Leica AF6000 fluorescence microscope.

For time-lapse video imaging, HeLa cells seeded onto chamber slides (Lab-TeK) were transfected with various expression constructs for $24 \mathrm{~h}$. The transfected cells were then cultured in a humidified chamber at $37^{\circ} \mathrm{C}$ in $\mathrm{CO}_{2}$-independent medium (GIBCO-BRL). GFP-positive cells were subject to time-lapse video imaging on a Leica TCS SP5 confocal microscope.

\section{ACKNOWLEDGMENTS AND FUNDING}

We thank Dr. Byeong Choi for valuable discussions during the course of the study. This work was supported in part by US Public Service Awards to WD (CA090658 
\& CA150512) and NIEHS Center grant (ES000260), CC

(CA100498 \& CA153354), and to LL (EY021797).

\section{CONFLICTS OF INTEREST}

No potential conflicts of interest are disclosed.

\section{REFERENCES}

1. Jia L, Kim S, Yu H. Tracking spindle checkpoint signals from kinetochores to $\mathrm{APC} / \mathrm{C}$. Trends in biochemical sciences. 2013; 38:302-311.

2. Ma HT, Poon RY. How protein kinases co-ordinate mitosis in animal cells. The Biochemical journal. 2011; 435:17-31.

3. Nigg EA. Mitotic kinases as regulators of cell division, its checkpoints. Nature reviews Molecular cell biology. 2001; 2:21-32.

4. Yu H. Cdc20: a WD40 activator for a cell cycle degradation machine. Molecular cell. 2007; 27:3-16.

5. Chao WC, Kulkarni K, Zhang Z, Kong EH, Barford D. Structure of the mitotic checkpoint complex. Nature. 2012; 484:208-213.

6. Sudakin V, Chan GK, Yen TJ. Checkpoint inhibition of the $\mathrm{APC} / \mathrm{C}$ in HeLa cells is mediated by a complex of BUBR1, BUB3, CDC20, and MAD2. The Journal of cell biology. 2001; 154:925-936.

7. Asteriti IA, Di Cesare E, De Mattia F, Hilsenstein V, Neumann B, Cundari E, Lavia P, Guarguaglini G. The Aurora-A inhibitor MLN8237 affects multiple mitotic processes and induces dose-dependent mitotic abnormalities and aneuploidy. Oncotarget. 2014; 5:6229-6242. doi:10.18632/oncotarget.2190.

8. Li J, Hong MJ, Chow JP, Man WY, Mak JP, Ma HT, Poon RY. Co-inhibition of polo-like kinase 1 and Aurora kinases promotes mitotic catastrophe. Oncotarget. 2015; 6: 9327-9340. doi:10.18632/oncotarget.3313.

9. Liu ST, Chan GK, Hittle JC, Fujii G, Lees E, Yen TJ. Human MPS1 kinase is required for mitotic arrest induced by the loss of CENP-E from kinetochores. Molecular biology of the cell. 2003; 14:1638-1651.

10. Stucke VM, Sillje HH, Arnaud L, Nigg EA. Human Mps1 kinase is required for the spindle assembly checkpoint but not for centrosome duplication. The EMBO journal. 2002; 21:1723-1732.

11. Hewitt L, Tighe A, Santaguida S, White AM, Jones CD, Musacchio A, Green S, Taylor SS. Sustained Mps1 activity is required in mitosis to recruit O-Mad2 to the Mad1-CMad2 core complex. The Journal of cell biology. 2010; 190:25-34.

12. Jelluma N, Brenkman AB, van den Broek NJ, Cruijsen CW, van Osch MH, Lens SM, Medema RH, Kops GJ. Mps1 phosphorylates Borealin to control Aurora B activity and chromosome alignment. Cell. 2008; 132:233-246.
13. Maciejowski J, George KA, Terret ME, Zhang C, Shokat KM, Jallepalli PV. Mps1 directs the assembly of Cdc20 inhibitory complexes during interphase and mitosis to control $\mathrm{M}$ phase timing and spindle checkpoint signaling. The Journal of cell biology. 2010; 190:89-100.

14. Maure JF, Kitamura E, Tanaka TU. Mps1 kinase promotes sister-kinetochore bi-orientation by a tension-dependent mechanism. Current biology. 2007; 17:2175-2182.

15. Santaguida S, Tighe A, D'Alise AM, Taylor SS, Musacchio A. Dissecting the role of MPS1 in chromosome biorientation and the spindle checkpoint through the small molecule inhibitor reversine. The Journal of cell biology. 2010; 190:73-87.

16. Sliedrecht T, Zhang C, Shokat KM, Kops GJ. Chemical genetic inhibition of Mps1 in stable human cell lines reveals novel aspects of Mps1 function in mitosis. PloS one. 2010; 5:e10251.

17. Herriott A, Sweeney M, Whitaker M, Taggart M, Huang JY. Kinetochore localized Mad2 and Cdc20 is itself insufficient for triggering the mitotic checkpoint when Mps1 is low in Drosophila melanogaster neuroblasts. Cell cycle. 2012; 11:4650-4660.

18. Dou Z, Sawagechi A, Zhang J, Luo H, Brako L, Yao XB. Dynamic distribution of TTK in HeLa cells: insights from an ultrastructural study. Cell research. 2003; 13:443-449.

19. Xu Q, Zhu S, Wang W, Zhang X, Old W, Ahn N, Liu X. Regulation of kinetochore recruitment of two essential mitotic spindle checkpoint proteins by Mps1 phosphorylation. Molecular biology of the cell. 2009; 20:10-20.

20. Palframan WJ, Meehl JB, Jaspersen SL, Winey M, Murray AW. Anaphase inactivation of the spindle checkpoint. Science. 2006; 313:680-684.

21. Stucke VM, Baumann C, Nigg EA. Kinetochore localization and microtubule interaction of the human spindle checkpoint kinase Mps1. Chromosoma. 2004; 113:1-15.

22. Sun T, Yang X, Wang W, Zhang X, Xu Q, Zhu S, Kuchta R, Chen G, Liu X. Cellular abundance of Mps1 and the role of its carboxyl terminal tail in substrate recruitment. The Journal of biological chemistry. 2010; 285:38730-38739.

23. Tatham MH, Hay RT. FRET-based in vitro assays for the analysis of SUMO protease activities. Methods Mol Biol. 2009; 497:253-268.

24. Cheng J, Kang X, Zhang S, Yeh ET. SUMO-specific protease 1 is essential for stabilization of HIF 1alpha during hypoxia. Cell. 2007; 131:584-595.

25. Gill G. SUMO and ubiquitin in the nucleus: different functions, similar mechanisms? Genes \& development. 2004; 18:2046-2059.

26. Bassi C, Ho J, Srikumar T, Dowling RJ, Gorrini C, Miller SJ, Mak TW, Neel BG, Raught B, Stambolic V. Nuclear PTEN controls DNA repair and sensitivity to genotoxic stress. Science. 2013; 341:395-399. 
27. Yang F, Yao Y, Jiang Y, Lu L, Ma Y, Dai W. Sumoylation is important for stability, subcellular localization, and transcriptional activity of SALL4, an essential stem cell transcription factor. The Journal of biological chemistry. 2012; 287:38600-38608.

28. Kang J, Chen Y, Zhao Y, Yu H. Autophosphorylationdependent activation of human Mps1 is required for the spindle checkpoint. Proceedings of the National Academy of Sciences of the United States of America. 2007; 104:20232-20237.

29. Dou Z, Liu X, Wang W, Zhu T, Wang X, Xu L, Abrieu A, $\mathrm{Fu}$ C, Hill DL, Yao X. Dynamic localization of Mps 1 kinase to kinetochores is essential for accurate spindle microtubule attachment. Proceedings of the National Academy of Sciences of the United States of America. 2015; 112: E4546-4555.

30. Lan W, Cleveland DW. A chemical tool box defines mitotic and interphase roles for Mps1 kinase. The Journal of cell biology. 2010; 190:21-24.

31. Morin V, Prieto S, Melines S, Hem S, Rossignol M, Lorca T, Espeut J, Morin N, Abrieu A. CDK-dependent potentiation of MPS1 kinase activity is essential to the mitotic checkpoint. Current biology: CB. 2012; 22: 289-295.

32. Wang W, Yang Y, Gao Y, Xu Q, Wang F, Zhu S, Old W, Resing K, Ahn N, Lei M, Liu X. Structural and mechanistic insights into Mps 1 kinase activation. Journal of cellular and molecular medicine. 2009; 13:1679-1694.

33. Jia H, Zhang X, Wang W, Bai Y, Ling Y, Cao C, Ma RZ, Zhong $\mathrm{H}$, Wang $\mathrm{X}, \mathrm{Xu} \mathrm{Q}$. A putative N-terminal nuclear export sequence is sufficient for Mps1 nuclear exclusion during interphase. BMC cell biology. 2015; 16:6.

34. Zhang X, Yin Q, Ling Y, Zhang Y, Ma R, Ma Q, Cao C, Zhong H, Liu X, Xu Q. Two LXXLL motifs in the N terminus of Mps1 are required for Mps1 nuclear import during $\mathrm{G}(2) / \mathrm{M}$ transition and sustained spindle checkpoint responses. Cell cycle. 2011; 10:2742-2750.

35. Nijenhuis W, von Castelmur E, Littler D, De Marco V, Tromer E, Vleugel M, van Osch MH, Snel B, Perrakis A, Kops GJ. A TPR domain-containing N-terminal module of MPS1 is required for its kinetochore localization by Aurora B. The Journal of cell biology. 2013; 201:217-231.

36. Cubenas-Potts C, Srikumar T, Lee C, Osula O, Subramonian D, Zhang XD, Cotter RJ, Raught B, Matunis MJ. Identification of SUMO-2/3-modified proteins associated with mitotic chromosomes. Proteomics. 2015; 15:763-772.
37. Yang F, Hu L, Chen C, Yu J, O'Connell CB, Khodjakov A, Pagano M, Dai W. BubR1 is modified by sumoylation during mitotic progression. The Journal of biological chemistry. 2012; 287:4875-4882.

38. Zhang XD, Goeres J, Zhang H, Yen TJ, Porter AC, Matunis MJ. SUMO-2/3 modification and binding regulate the association of CENP-E with kinetochores and progression through mitosis. Molecular cell. 2008; 29:729-741.

39. Conde C, Osswald M, Barbosa J, Moutinho-Santos T, Pinheiro D, Guimaraes S, Matos I, Maiato H, Sunkel CE. Drosophila Polo regulates the spindle assembly checkpoint through Mps1-dependent BubR1 phosphorylation. The EMBO journal. 2013; 32:1761-1777.

40. Elowe S, Hummer S, Uldschmid A, Li X, Nigg EA. Tension-sensitive Plk1 phosphorylation on BubR1 regulates the stability of kinetochore microtubule interactions. Genes \& development. 2007; 21:2205-2219.

41. Jelluma N, Dansen TB, Sliedrecht T, Kwiatkowski NP, Kops GJ. Release of Mps1 from kinetochores is crucial for timely anaphase onset. The Journal of cell biology. 2010; 191:281-290.

42. Yang F, Huang Y, Dai W. Sumoylated BubR1 plays an important role in chromosome segregation and mitotic timing. Cell cycle. 2012; 11:797-806.

43. Daniel J, Coulter J, Woo JH, Wilsbach K, Gabrielson E. High levels of the Mps1 checkpoint protein are protective of aneuploidy in breast cancer cells. Proceedings of the National Academy of Sciences of the United States of America. 2011; 108:5384-5389.

44. Janssen A, Kops GJ, Medema RH. Elevating the frequency of chromosome mis-segregation as a strategy to kill tumor cells. Proceedings of the National Academy of Sciences of the United States of America. 2009; 106:19108-19113.

45. Fisk HA, Mattison CP, Winey M. Human Mps1 protein kinase is required for centrosome duplication and normal mitotic progression. Proceedings of the National Academy of Sciences of the United States of America. 2003; 100:14875-14880.

46. Pike AN, Fisk HA. Centriole assembly and the role of Mps 1: defensible or dispensable? Cell division. 2011; 6:9.

47. Majumder S, Slabodnick M, Pike A, Marquardt J, Fisk HA. VDAC3 regulates centriole assembly by targeting Mps1 to centrosomes. Cell cycle. 2012; 11:3666-3678.

48. Kasbek C, Yang CH, Fisk HA. Mps1 as a link between centrosomes and genomic instability. Environmental and molecular mutagenesis. 2009; 50:654-665. 